\title{
Bioinformatics-Based Identification of a circRNA-miRNA-mRNA Axis in Esophageal Squamous Cell Carcinomas
}

\author{
Zhaojun Wang, ${ }^{1}$ Haifeng $\mathrm{Li}^{2}{ }^{2}$ Fajun $\mathrm{Li}^{3}{ }^{3}$ Xin $\mathrm{Su},{ }^{4}$ and Junhang Zhang $\mathbb{D}^{1}$ \\ ${ }^{1}$ Department of Thoracic Surgery, The Seventh Affiliated Hospital, Sun Yat-sen University, Shenzhen, China \\ ${ }^{2}$ Department of Anesthesiology, Guangdong General Hospital, Guangzhou, China \\ ${ }^{3}$ Department of Critical Care Medicine, The First People's Hospital of Kunshan, Kunshan, China \\ ${ }^{4}$ Department of Respiratory, Hainan Hospital of PLA General Hospital, Sanya, China \\ Correspondence should be addressed to Junhang Zhang; zhangjunhang1111@163.com
}

Received 17 August 2020; Revised 16 September 2020; Accepted 17 September 2020; Published 29 September 2020

Academic Editor: Nicola Silvestris

Copyright (c) 2020 Zhaojun Wang et al. This is an open access article distributed under the Creative Commons Attribution License, which permits unrestricted use, distribution, and reproduction in any medium, provided the original work is properly cited.

\begin{abstract}
Background. Esophageal squamous cell carcinoma (ESCC) has a poor prognosis due to the lack of early disease symptoms. Using bioinformatics tools, this study aimed to discover differentially expressed nonprotein-coding RNAs and genes with potential prognostic relevance in ESCC. Methods. Two microRNAs (miRNAs) and one circular RNA (circRNA) microarray datasets were downloaded from the Gene Expression Omnibus (GEO) database. Differential expression of miRNAs (DEMs) and circRNAs (DECs) was, respectively, identified in ESCC tissue and compared to adjacent healthy tissue. Further analysis was performed using the miRNA microarray datasets, where miRTarBase was used to predict which messenger RNAs (mRNAs) was present. This was followed by protein-protein interaction (PPI) network, Kyoto Encyclopedia of Genes and Genomes (KEGG), and Gene Ontology (GO) analyses. Moreover, cytoHubba and UALCAN were used to predict the important nodes and perform patient survival analysis, respectively. The miRNA-associated circRNAs were predicted using the ENCORI website. The interaction between DECs and the predicted circRNAs was also determined. A circRNA-miRNA-mRNA axis was constructed. Results. Associated with $R A P 1 B$ and circ_0052867, two miRNAs (miR-133b and miR-139-5p) were identified as being differentially expressed and downregulated across the two datasets. Finally, the circ_0052867/miR-139-5p/RAP1B regulatory axis was established. Conclusion. This study provides support for the possible mechanisms of disease progression in ESCC.
\end{abstract}

\section{Introduction}

Globally, esophageal carcinoma is the eighth most common type of cancer, and the sixth most dominant cause of cancer death $[1,2]$. Esophageal squamous cell carcinoma (ESCC) is the major pathology (60-70\%) of esophageal cancer (ESCA) [3]. Despite great advances in surgical and radiation therapy, the five-year survival rate of ESCC patients is still under 25\%. This is because obvious clinical symptoms of early-stage ESCA are lacking, and many patients are therefore already in an advanced disease stage at the time of diagnosis [3]. A better prognosis is related to the early diagnosis of disease [4]. While molecular markers have been regarded as prognostic markers of ESCC in recent years $[5,6]$, these biomarkers are not always applicable for early diagnosis or prognosis prediction. Therefore, there is an urgent need to identify new biomarkers to improve the diagnosis and prognosis of ESCC. Biomarkers are stably expressed in plasma, serum, and other body fluids and include circRNAs, miRNAs, and genes [7].

The occurrence and progression of ESCC are related to genetic factors such as genomic amplifications, mutations, insertions, and deletions, as well as tumor epigenetics. The latter includes noncoding RNA, DNA methylation, and histone acetylation $[7,8]$. MiRNAs are endogenous, singlestranded, noncoding RNA polymers formed from 20-22 nucleotides and encoded by a single nuclear DNA. They are mainly involved in transcriptional and posttranscriptional regulation of gene expression and bind to complementary sequences of target mRNA. Decreased expression of tumor suppressor miRNA-associated genes and overexpression of oncogenic miRNAs may lead to tumor formation [9]. 
Recently, miRNAs have been accepted as good prognostic or diagnostic biomarkers in cancer research [10]. Several studies have assessed the possible role of miRNA in identifying the potential for hepatocellular carcinoma metastasis and tumor recurrence [11], and it has also been shown that miRNAs can act on different pathways associated with chemotherapeutic drug sensitivity or resistance in several tumors $[12,13]$. Sun et al. found that the level of serum miRNA-1290 could be a useful diagnostic and prognostic marker in ESCC patients [14]. Similarly, miR-455-3p was found to play an antioncogenic role by downregulating $F A M 83 F$ and could be an independent clinical biomarker for predicting the outcome of ESCC patients [15]. One study indicated that decreased miR-145 expression levels could be a useful prognostic marker and could be used to predict overall survival in ESCC patients [16]. The ciRS-7 molecule was shown to be a novel prognostic biomarker and therapeutic target for ESCC [17].

This study aimed to identify differentially expressed miRNAs (DEMs) using GEO datasets, predict which mRNAs were associated to the DEMs, and perform functional enrichment and protein-protein interaction (PPI) network analysis to detect potential disease-associated target genes. The key ESCC-associated genes were identified by survival analysis. The intersection between predicted miRNAs, circRNAs, and differentially expressed circRNAs (DECs) was determined against GEO data, and a potential circRNA-miRNA-mRNA network for ESCC was subsequently established.

\section{Materials and Methods}

2.1. Source of the Microarray Data. The public GEO database (http://www.ncbi.nlm.nih.gov/geo) is a functional genomic database from which three ESCC microarray expression datasets (GSE97051, GSE59973, and GSE131969) were downloaded. The circRNA dataset (GSE131969) included data from three ESCC tissue and three adjacent noncancerous tissue samples. The samples were tested against GPL19978 Agilent-069978 Arraystar Human CircRNA microarray V1 data. The miRNA dataset (GSE97051) included data from seven ESCC tissue and seven nonpathogenic esophageal tissue samples that were tested against GPL21572 Agilent-067406 Human CBC lncRNA + mRNA microarray V4.0 data. The miRNA dataset (GSE59973) included three ESCC tissue and three nonpathogenic esophageal tissue samples that were tested against data from the GPL16770 Agilent-031181 Unrestricted_Human_miRNA_V16.0_microarray system.

\subsection{Identification of DECs and DEMs. Differential analysis} was conducted for GSE97051, GSE59973, and GSE131969 to obtain DEMs and DECs between ESCC and healthy control tissue samples by using GEO2R (http://www.ncbi.nlm.nih. gov/geo/geo $2 \mathrm{r} /$ ) [18]. The interaction across miRNA microarray datasets was performed through Venny (http:// bioinformatics.psb.ugent.be/webtools/Venn/). Using ENCORI (http://starbase.sysu.edu.cn/index.php), which is an opensource platform for studying RNA interactomes [19], the expression and overall survival of DEMs was examined. The criterion for DECs was an adjusted $p$ value $<0.05$ and $|\log \mathrm{FC}| \geqq 2$, while the criterion for DEMs was an adjusted $p$ value $<0.05$ and $|\log \mathrm{FC}| \geqq 1$. Volcano plots of the DEMs were created using Sangerbox (http://sangerbox.com/ AllTools?tool_id=9699135). The miRTarBase was used to predict potentially significant mRNAs (http://mirtarbase. cuhk.edu.cn/php/index.php).

2.3. Functional Enrichment Analysis of Differentially Expressed Genes. GO and KEGG were used to identify the gene functions of individual genomic products and associated pathway information. GO evaluated the cellular component (CC), biological process (BP), and molecular function (MF) of each element $[20,21]$. Annotation, visualization, and integrated discovery were performed using the online DAVID database tool (https://david. ncifcrf.gov/). A $p$ value $<0.05$ was considered to be significant.

2.4. Construction of Protein-Protein Interaction (PPI) Networks and Validation of the Hub Genes. The STRING database was applied for PPI network construction. Using Cytoscape via the CytoHubba app, the top 50 candidates were considered to be hub genes. To determine the expression of the 50 hub genes in ESCC tissue and to understand the effect(s) of hub genes on prognosis, the expression and survival analyses of hub genes were performed using UALCAN (http://ualcan.path.uab.edu/index. html). The miRNA-mRNA subnetwork of interest was determined. $p<0.05$ was considered to indicate statistical significance.

2.5. Validation of the circRNAs and Construction of the Competitive Endogenous RNA Network. StarBase 3.0 was introduced to predict linkage between the miRNAs and circRNAs. Using Venny against the GEO dataset, DECs of interest were predicted. The intersection between circRNAs and these DECs indicated the target circRNAs in ESCC tissue. Finally, a circRNA-miRNA-mRNA network was constructed, and the data were visualized using Cytoscape software 3.7.1.

\section{Results}

3.1. Two Potential miRNAs Selected for ESCC. To find the potential miRNA biomarkers in ESCC, two miRNA datasets (GSE97051 and GSE59973) were selected (Table 1). As per the selection criteria, and as shown in Figure 1(d), two downregulated DEMs (miR-133b and miR-139-5p) were isolated. Next, the expression and overall survival of two downregulated DEMs in ESCA data were examined using ENCORI. Both miR-133b $(p=0.01)$ and miR-139-5p $(p=6.6 e-8)$ showed significantly low expression in cancer samples, but no effect on overall patient survival was seen in ESCA (Figure 2). 
TABLE 1: Basic information of the three microarray datasets from GEO.

\begin{tabular}{lccc}
\hline Data source & Platform & Series & Sample size (T/N) \\
\hline circRNA & GPL19978 & GSE131969 & $3 / 3$ \\
miRNA & GPL16770 & GSE59973 & $3 / 3$ \\
miRNA & GPL21572 & GSE97051 & $7 / 7$ \\
\hline
\end{tabular}

Note. T: tumor; $\mathrm{N}$ : normal.

3.2. Prediction and Analysis of $m R N A$ s Binding to the Potential ESCC-Associated miRNAs. The miRTarBase database was used to identify which mRNAs might bind to the two predicted miRNAs. In total, 87 mRNAs potentially bind to miR-133b, and 105 mRNAs potentially bind to miR-139-5p. In order to identify key nodes in the PPI network, the top 50 hub genes were selected using CytoHubba based on the STRING database (Figure 3(c) and Table S1). For better visualization, the miRNA-mRNA connecting network was constructed using Cytoscape (Figure 3(d)).

3.3. Enrichment Analysis of the Target Genes. KEGG enrichment and GO analyses were performed for the target genes of the two DEMs (Figure 2). In the BP category, the "extrinsic apoptotic signaling pathway in absence of ligand," "negative regulation of anoikis," and "negative regulation of apoptotic process" were enriched. While "protein binding" was enriched according to the MF category, enrichment of the "nucleoplasm," "nucleus," and "cytosol" was shown in the CC category (Figure 3(b)). The KEGG pathway analysis revealed enrichment of the "focal adhesion," "estrogen signaling," "sphingolipid signaling," and "PI3K-Akt signaling" pathways (Figure 3(a)).

3.4. Identification of mRNA Influences Patient Survival. To analyze the 50 mRNAs selected by the PPI network, survival analysis was performed using UALCAN. It was found that only $R A P 1 B$ expression was significantly increased in ESCA tumor samples $(p=2.3529 e-4)$. Additionally, the higher the expression of $R A P 1 B$, the worse the patient's survival $(p=0.011)$. The miR-139-5p/RAP1B subnetwork was therefore determined.

3.5. Validation of the circRNAs and Construction of the Competitive Endogenous RNA Network. ENCORI was utilized to identify the 3766 potential circRNAs targeted by miR-139-5p. The intersection of these circRNAs and the predicted DECs found circ_0052867 to be of interest (Figure 4(c)). Finally, the circ_0052867/miR-139-5p/RAP1B network was constructed (Figure 4(d)) and was concluded to be a circRNA-miRNA-mRNA regulatory axis.

\section{Discussion}

Due to the absence of obvious clinical symptoms in the early stages of ESCC and the lack of sensitive early diagnostic detection methods, most patients with ESCC are diagnosed at an advanced disease stage. Consequently, the best treatment opportunity is lost. Stein et al. reported that the fiveyear survival rate for early ESCA was $62.9 \%$ following surgery [22]. Nevertheless, the validity and sensitivity in detecting traditional tumor markers are insufficient for early ESCC diagnoses [23]. There is therefore an urgent need for improved biomarkers for early detection of ESCC.

Playing an important role in pathological and physiological processes, noncoding RNA comprises approximately $98 \%$ of the human genome [24]. Many studies have demonstrated that competitive endogenous (ceRNAs) are able to act as sponges for miRNAs [25], while miRNAs have been shown to play an important role in tumorigenesis and its prognosis [26]. Li et al. found that the survival time of high miRNA-506 expression was significantly shorter than that of low miRNA-506 expression [27]. Downregulated miRNA718 expression may also serve as a potential diagnostic marker for ESCC [28].

The circRNAs are newly found noncoding RNAs that participate in cancer development [29] and are currently being investigated as potential cancer biomarkers [30,31]. Leading to poor prognosis in ESCC, upregulated circRNA_100876 expression can accelerate cell proliferation and metastasis [32], while the upregulation of circRNA_100873 is associated with increased lymphatic metastasis in ESCC [33]. A further study reported that circGSK3 overexpression enhances multiple metastasis and invasion of the tumor [34]. The underlying mechanisms of circRNAs and miRNAs in ESCC patient survival remain unknown.

Real-time quantitative PCR (q-PCR) is a classical method to detect abnormal noncoding RNA expression in ESCC tissue. Next generation sequencing techniques and available microarray data provide researchers with a more complete noncoding RNA expression profile between ESCC tumor and nonpathogenic tissue samples. For example, Yang et al. detected 39 dysregulated miRNAs (28 downregulated and 11 upregulated) in tumor/nonpathogenic ESCC tissue [35]. Serum miRNA expression profiles were detected in 52 ESCC patients, and three miRNAs were identified as biomarkers for the diagnosis of ESCC [36].

In the present study, the intersection between two GEO databases confirmed miR-133b and miR-139-5p to be of interest, with an miRNA-mRNA-circRNA interaction being predicted. According to ceRNA theory, the expressions of miRNAs should be negatively correlated with the expressions of its targeted mRNAs and circRNAs. In this study, two downregulated DEMs were predicted to be associated with upregulated mRNA and circRNA targets. The PPI network was used to identify hub genes, and survival analysis was performed in order to find the key genes. Subsequently, $R A P 1 B$ and circ_0052867 were found to have an impact on the survival of ESCC patients. Consequently, we constructed the circ_0052867/miR-139-5p/RAP1B ceRNA regulatory 

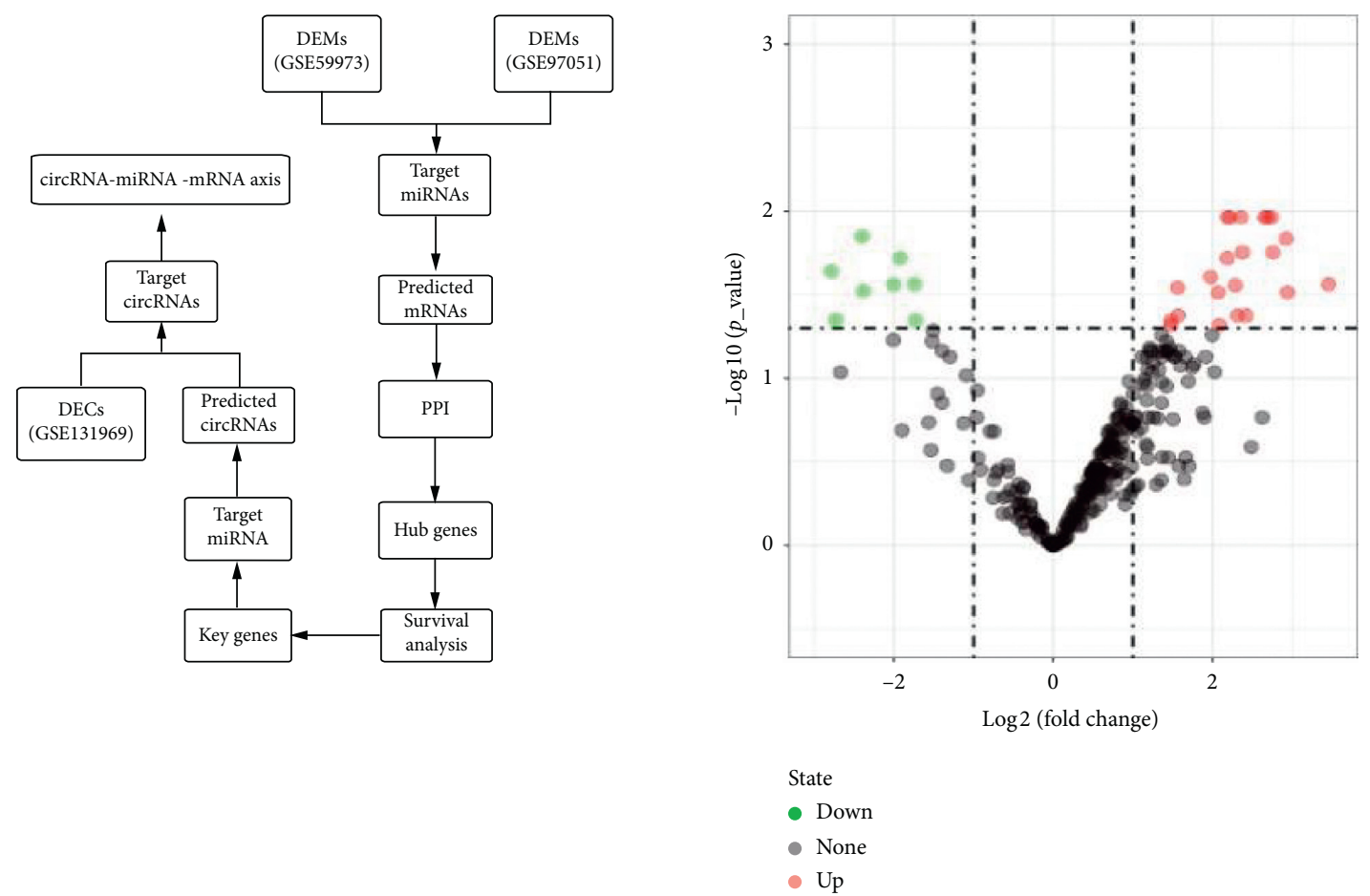

(a)
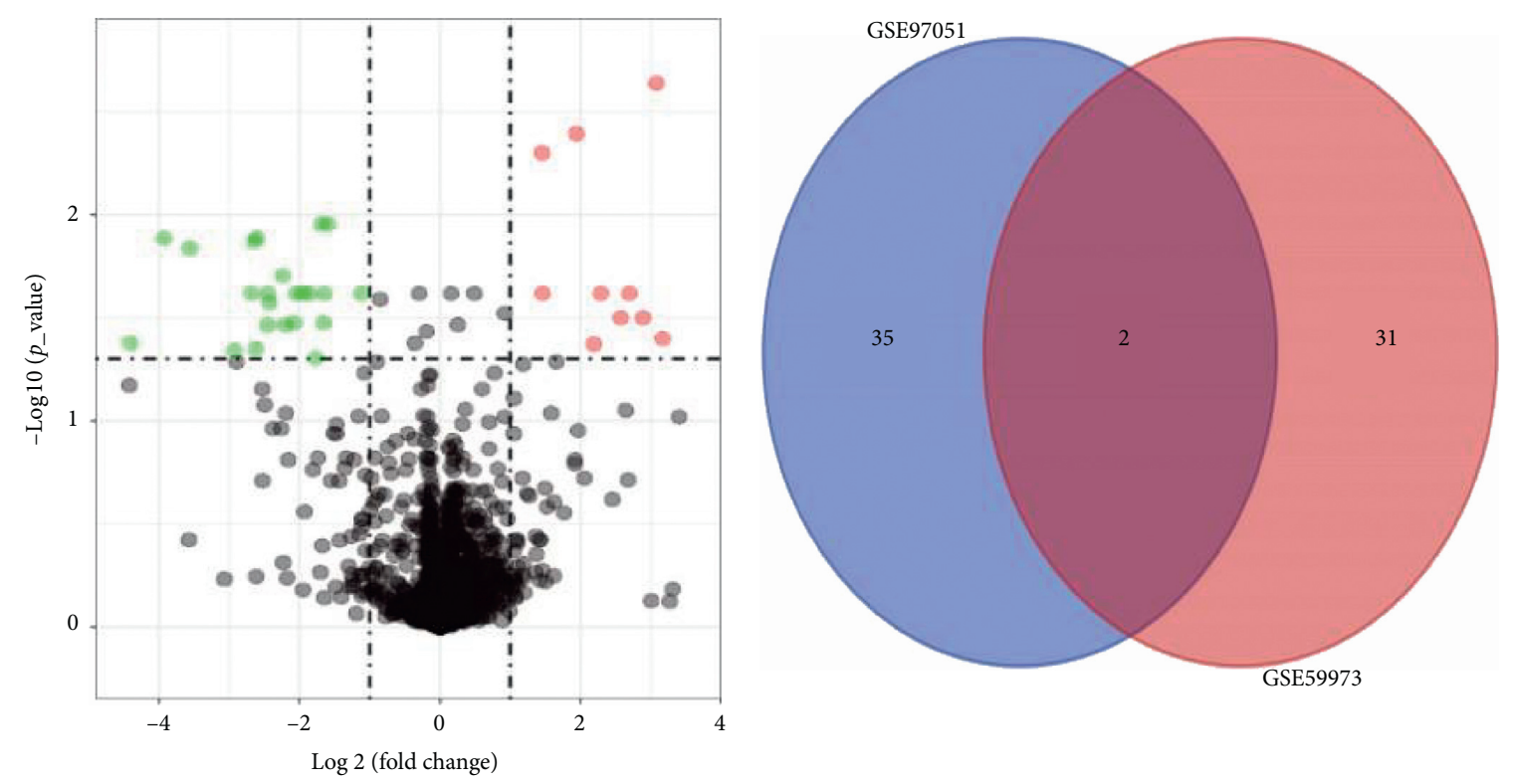

State

- Down

- None

- Up

(c)

(d)

FIGURE 1: Flow chart of the approach and identification of potential miRNAs in ESCA (esophageal cancer). (a) Flow chart of the approach utilized in this study. DEMs: differential expression of miRNAs and DECs: differential expression of circRNAs. (b) The volcano plot of differentially expressed miRNAs from GSE59973 dataset. (c) The volcano plot of differentially expressed miRNAs from GSE97051 dataset. (d) The intersection analysis of DEMs from GSE59973 dataset and GSE97051 dataset.

network, which may be a potential biomarker for ESCC patient survival. To our knowledge, this is the first study to report the expression of circ_0052867 in ESCC. While it has been found that the expression level of miR-139-5P in 11 cases of esophageal cancer is significantly higher than that of adjacent paracarcinoma tissue [37]. Yang et al. reported that 


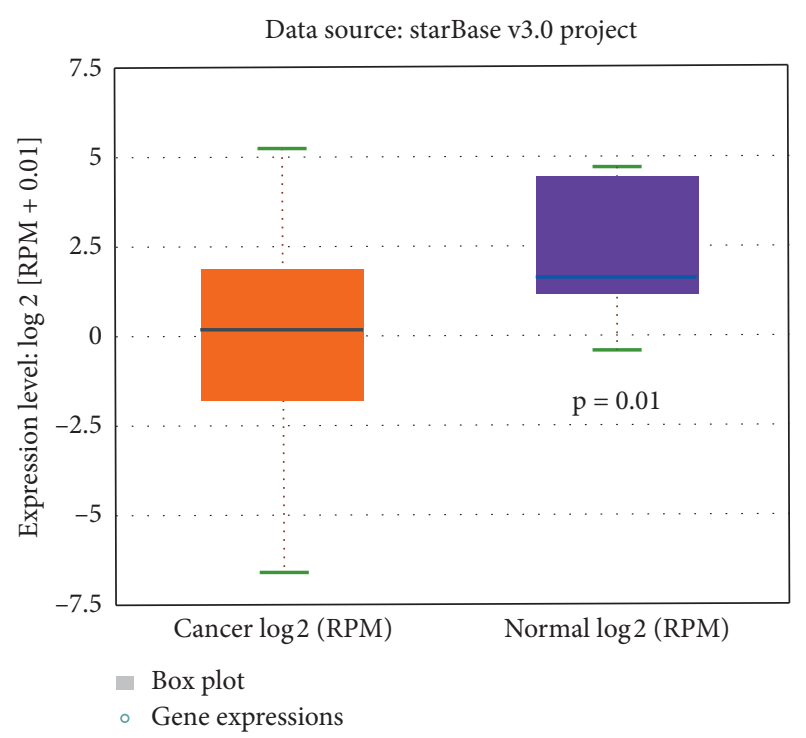

(a)

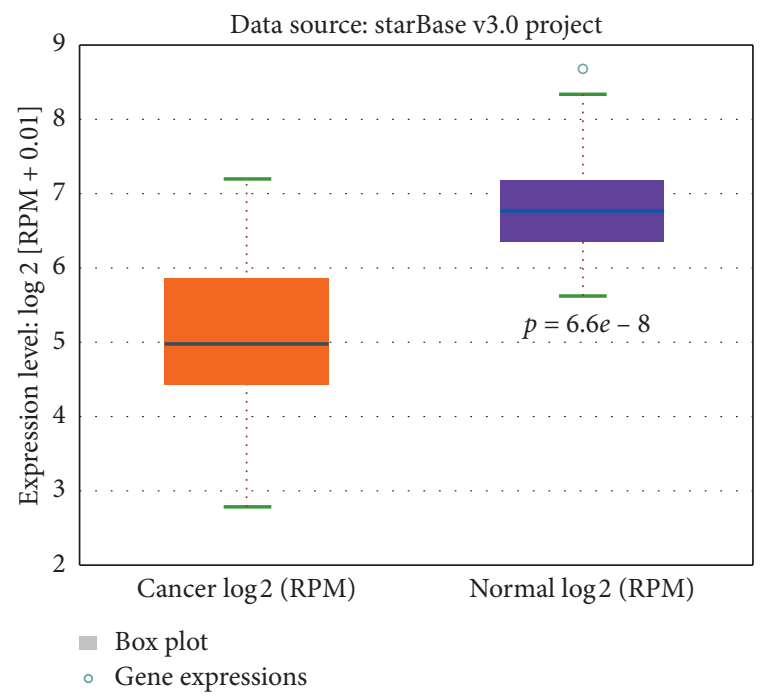

(c)

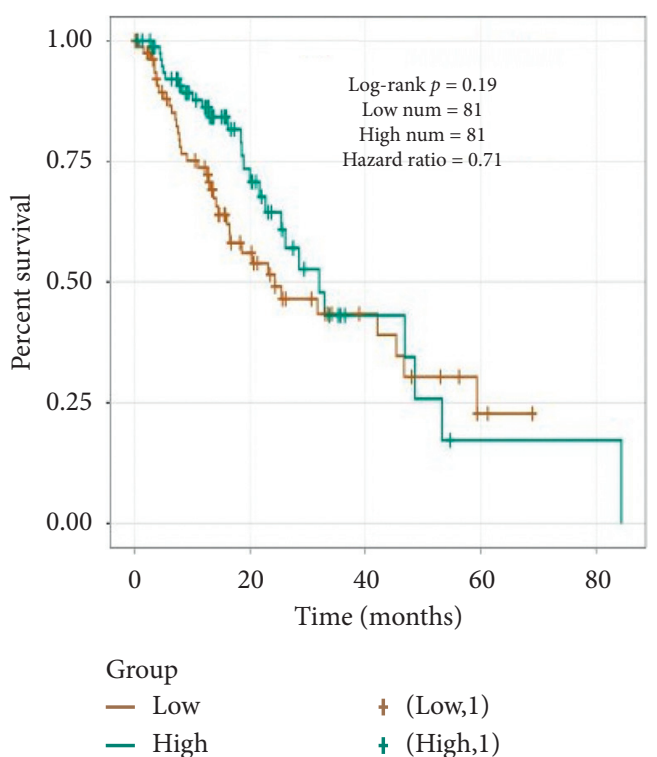

(b)

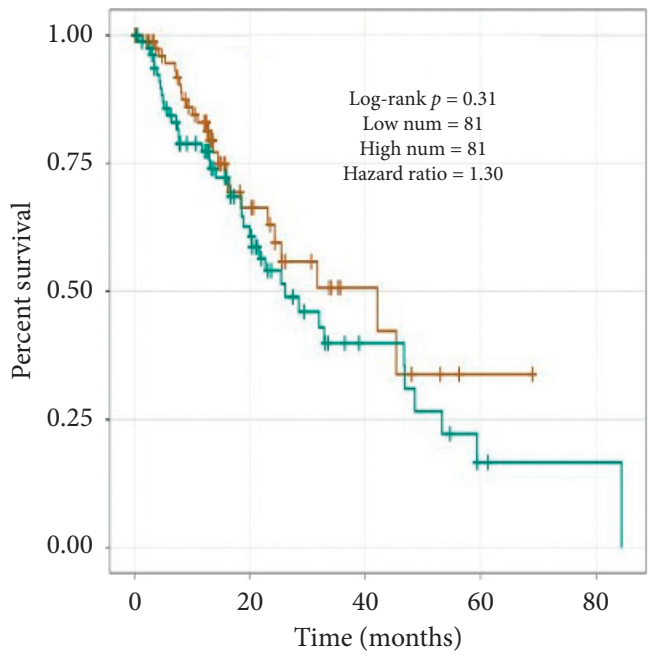

$$
\begin{array}{ll}
\text { Group } & \\
\text { - Low } & +(\text { Low, } 1) \\
\text { - High } & + \text { (High, } 1)
\end{array}
$$

(d)

FIGURE 2: The expression level and overall survival of miRNAs in ESCA (esophageal cancer). (a) The expression level of hsa-miR-133b with 162 cancer and 11 normal samples in ESCA. (b) Overall survival for hsa-miR-133b in ESCA cancer. (c) The expression level of hsa-miR-139$5 p$ with 162 cancer and 11 normal samples in ESCA. (d) Overall survival for hsa-miR-139-5p in ESCA cancer. $p<0.05$ represents significant difference.

hsa-miR-139-5p was downregulated in ESCC tumor tissue compared to nontumor tissue samples [38]. Jia et al. found that the expression of RAP1B was upregulated in ESCC tissue, and $R A P 1 B$ promoted the growth, migration, and metastasis of the ESCC cells [39]. However, Zhang et al. concluded that miR-518b may play an anticancer role in the development of ESCC by targeting RAP1B [40]. Nevertheless, the exact mechanism remains to be discovered.
There were still some limitations to this study. Firstly, the findings of this study require further experimental verification. Secondly, the sample size is too small, and there are only a limited number of samples in each microarray dataset. Lastly, as mRNAs that influence patient survival and miRNAs that alter expression in ESCC tissue were identified in a database which includes ESCA tumor samples, there may be bias towards ESCC patients in this analysis. 


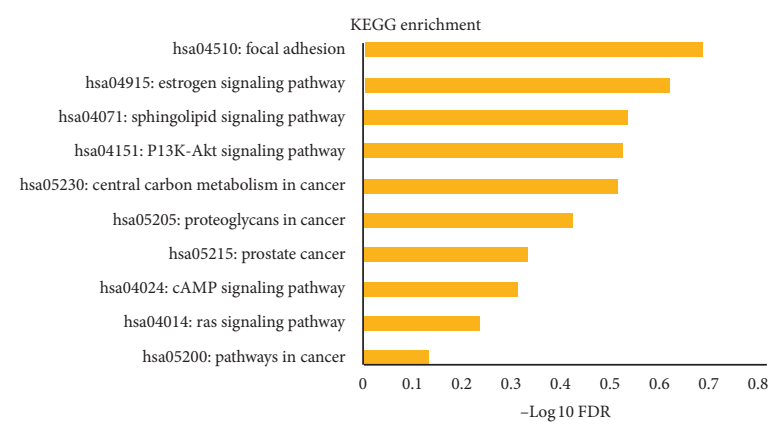

(a)

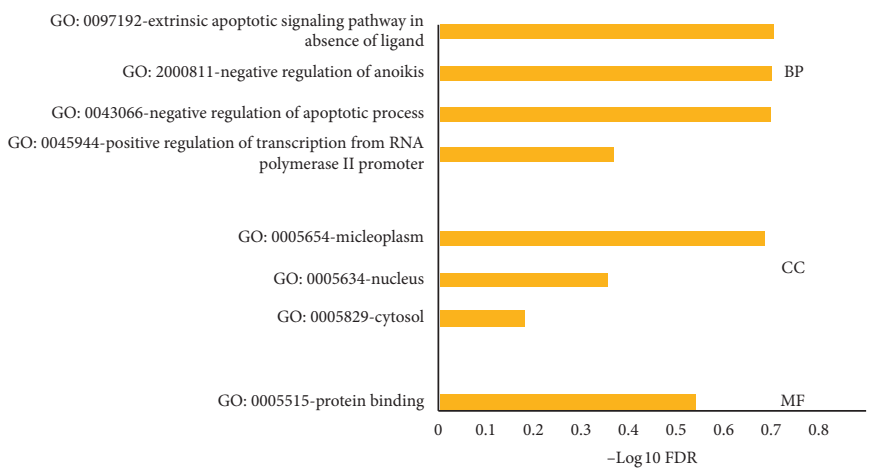

(b)

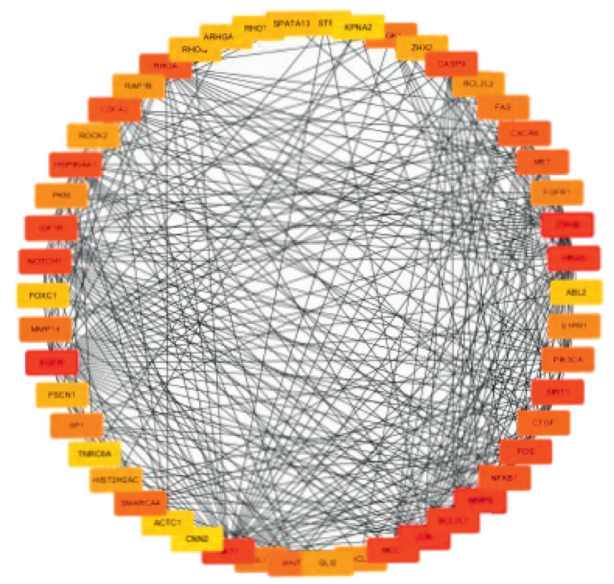

(c)

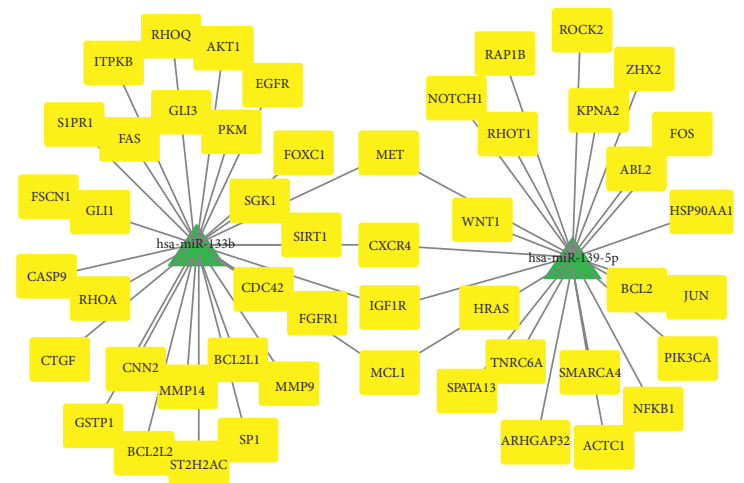

(d)

FIGURE 3: Enrichment analysis of target genes, identification of hub genes, and the network of miRNA-target genes. (a) KEGG analyses. (b) GO analyses. (c) The top 50 hub genes were selected CytoHubba. (d) The network of miRNA-target genes. 


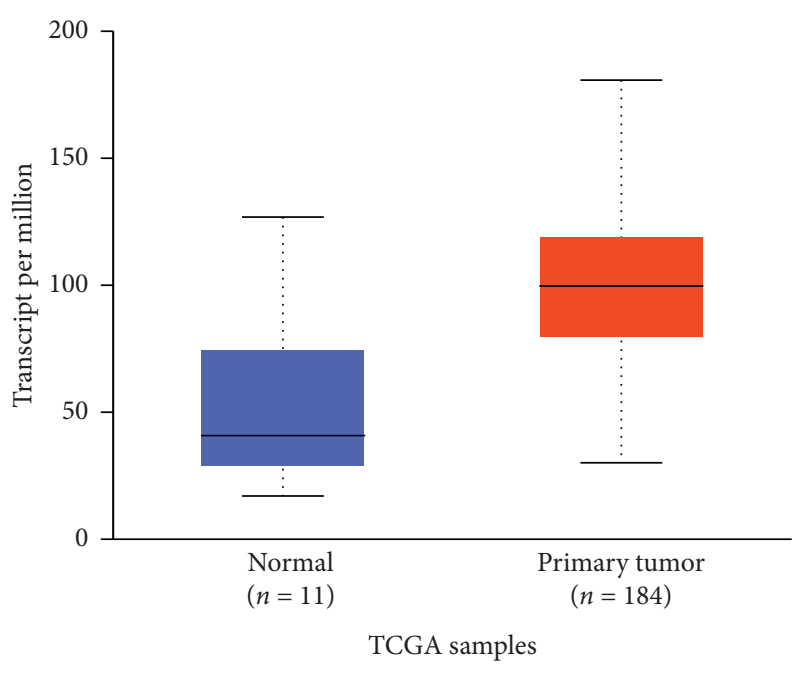

(a)

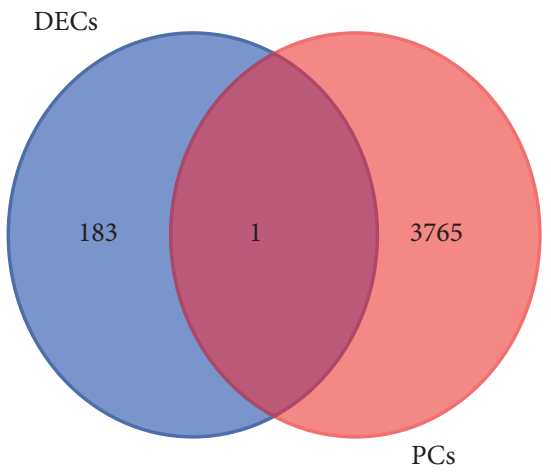

(c)

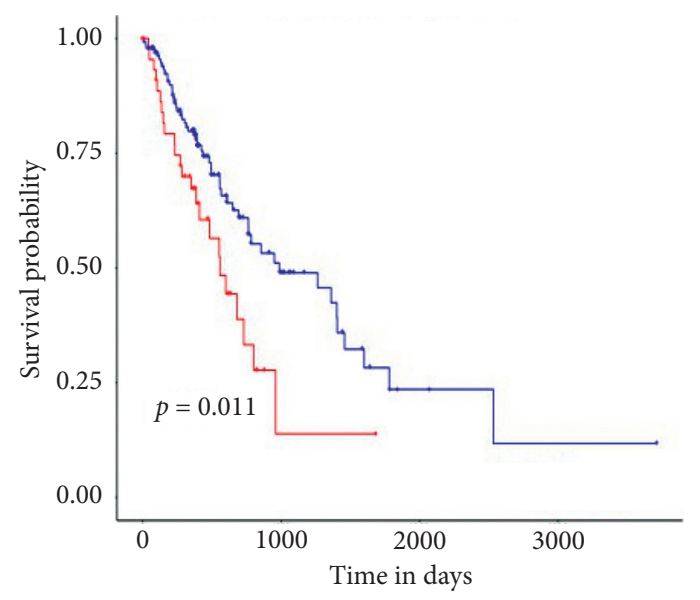

Expression level

$\Delta$ High expression $(n=46)$

- Low medium-expression $(n=138)$

(b)

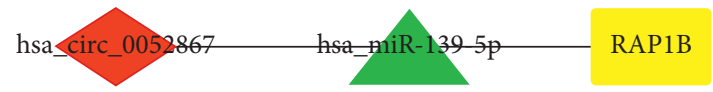

Figure 4: The network of circRNA-miRNA-target genes. (a) The expression level of RAP1B in ESCA based on sample type. (b) Effect of $R A P 1 B$ expression level on ESCA patient survival. (c) The intersection analysis of DECs and PCs. DECs: differential expression of circRNAs and PCs: predicted circRNAs. (d) The network of circRNA-miRNA-target genes axis. $p<0.05$ represents significant difference.

\section{Data Availability}

The data used to support this study are available in TCGA database and GEO database.

\section{Conflicts of Interest}

The authors declare no conflicts of interest in this work.

\section{Authors' Contributions}

Zhaojun Wang wrote the manuscript. Junhang Zhang conceived the study and provided guidance. All other authors contributed to the analysis and reviewed the manuscript. Zhaojun Wang and Haifeng Li contributed equally to this work.

\section{Acknowledgments}

The authors thank International Science Editing (http:// www.internationalscienceediting.com) for editing this manuscript.

\section{Supplementary Materials}

Table S1: 50 hub genes selected using CytoHubba ranked by MCC method. (Supplementary Materials)

\section{References}

[1] W. Chen, R. Zheng, P. D. Baade et al., "Cancer statistics in China, 2015," CA: A Cancer Journal for Clinicians, vol. 66, no. 2, pp. 115-132, 2016.

[2] R. Siegel, J. Ma, Z. Zou, and A. Jemal, "Cancer statistics, 2014," CA: A Cancer Journal for Clinicians, vol. 64, no. 1, pp. 9-29, 2014.

[3] M. Maghsudlu, E. Farashahi Yazd, and T. Amiriani, "Increased expression of MiR-27a and MiR-24-2 in esophageal squamous cell carcinoma," Journal of Gastrointestinal Cancer, vol. 51, no. 1, pp. 227-233, 2020.

[4] A. Pennathur, M. K. Gibson, B. A. Jobe, and J. D. Luketich, "Oesophageal carcinoma," The Lancet, vol. 381, no. 9864, pp. 400-412, 2013.

[5] W. Cao, W. Wu, F. Shi et al., "Integrated analysis of long noncoding RNA and coding RNA expression in esophageal 
squamous cell carcinoma," International Journal of Genomics, vol. 2013, Article ID 480534, 10 pages, 2013.

[6] S. Mehta, A. Shelling, A. Muthukaruppan et al., "Predictive and prognostic molecular markers for cancer medicine," Therapeutic Advances in Medical Oncology, vol. 2, no. 2, pp. 125-148, 2010.

[7] C.-Y. Li, W.-W. Zhang, J.-L. Xiang, X.-H. Wang, J. Li, and J.-L. Wang, "Identification of microRNAs as novel biomarkers for esophageal squamous cell carcinoma: a study based on the cancer genome atlas (TCGA) and bioinformatics," Chinese Medical Journal, vol. 132, no. 18, pp. 2213-2222, 2019.

[8] C. Kandoth, M. D. Mclellan, F. Vandin et al., "Mutational landscape and significance across 12 major cancer types," Nature, vol. 502, no. 7471, pp. 333-339, 2013.

[9] O. Brunetti, A. Russo, A. Scarpa et al., "MicroRNA in pancreatic adenocarcinoma: predictive/prognostic biomarkers or therapeutic targets?" Oncotarget, vol. 6, no. 27, pp. 23323-23341, 2015.

[10] F. R. Yang, H. J. Li, T. T. Li et al., "Prognostic value of microrna-15a in human cancers: a meta-analysis and bioinformatics," BioMed Research International, vol. 2019, Article ID 2063823, 12 pages, 2019.

[11] A. Gnoni, D. Santini, M. Scartozzi et al., "Hepatocellular carcinoma treatment over sorafenib: epigenetics, microRNAs and microenvironment. Is there a light at the end of the tunnel?" Expert Opinion on Therapeutic Targets, vol. 19, no. 12, pp. 1623-1635, 2015

[12] K. Danza, N. Silvestris, G. Simone et al., "Role of miR-27a, miR-181a and miR-20b in gastric cancer hypoxia-induced chemoresistance," Cancer Biology \& Therapy, vol. 17, no. 4, pp. 400-406, 2016.

[13] R. Pinto, S. Strippoli, S. De Summa et al., "MicroRNA expression in BRAF-mutated and wild-type metastatic melanoma and its correlation with response duration to BRAF inhibitors," Expert Opinion on Therapeutic Targets, vol. 19, no. 8, pp. 1027-1035, 2015.

[14] H. Sun, L. Wang, Q. Zhao, and J. Dai, "Diagnostic and prognostic value of serum miRNA-1290 in human esophageal squamous cell carcinoma," Cancer Biomarkers, vol. 25, no. 4, pp. 381-387, 2019.

[15] H. Yang, Y. N. Wei, J. Zhou et al., "MiR-455-3p acts as a prognostic marker and inhibits the proliferation and invasion of esophageal squamous cell carcinoma by targeting FAM83F," European Review for Medical and Pharmacological Sciences, vol. 21, no. 14, pp. 3200-3206, 2017.

[16] W. Jin, W. Luo, W. Fang et al., "miR-145 expression level in tissue predicts prognosis of patients with esophageal squamous cell carcinoma," Pathology-Research and Practice, vol. 215, no. 6, Article ID 152401, 2019.

[17] R. C. Li, S. Ke, F. K. Meng et al., "CiRS-7 promotes growth and metastasis of esophageal squamous cell carcinoma via regulation of miR-7/HOXB13," Cell Death \& Disease, vol. 9, no. 8, Article ID 838, 2018.

[18] Y. Qin, L. Li, F. Wang et al., "Knockdown of mir-135b sensitizes colorectal cancer cells to oxaliplatin-induced apoptosis through increase of FOXO1," Cellular Physiology and Biochemistry, vol. 48, no. 4, pp. 1628-1637, 2018.

[19] W. Wang, W. Lou, B. Ding et al., "A novel mRNA-miRNAlncRNA competing endogenous RNA triple sub-network associated with prognosis of pancreatic cancer," Aging, vol. 11, no. 9, pp. 2610-2627, 2019.

[20] M. Ashburner, C. A. Ball, J. A. Blake et al., "Gene ontology: tool for the unification of biology. The gene ontology consortium," Nature Genetics, vol. 25, no. 1, pp. 25-29, 2000.
[21] J. Du, Z. Yuan, Z. Ma, J. Song, X. Xie, and Y. Chen, "KEGGPATH: kyoto encyclopedia of genes and genomes-based pathway analysis using a path analysis model," Molecular BioSystems, vol. 10, no. 9, pp. 2441-2447, 2014.

[22] H. J. Stein, M. Feith, B. L. Bruecher, J. Naehrig, M. Sarbia, and J. R. Siewert, "Early esophageal cancer: pattern of lymphatic spread and prognostic factors for long-term survival after surgical resection," Annals of Surgery, vol. 242, pp. 566-573, 2005.

[23] H. Zhang, H. Li, Q. Ma, F.-Y. Yang, and T.-Y. Diao, "Predicting malignant transformation of esophageal squamous cell lesions by combined biomarkers in an endoscopic screening program," World Journal of Gastroenterology, vol. 22, no. 39, pp. 8770-8778, 2016.

[24] Y. Zhang, Y. Xu, L. Feng et al., "Comprehensive characterization of IncRNA-mRNA related ceRNA network across 12 major cancers," Oncotarget, vol. 7, no. 39, pp. 64148-64167, 2016.

[25] W.-H. Xue, Z.-R. Fan, L.-F. Li et al., "Construction of an oesophageal cancer-specific ceRNA network based on miRNA, lncRNA, and mRNA expression data," World Journal of Gastroenterology, vol. 24, no. 1, pp. 23-34, 2018.

[26] S. Zhu, M.-L. Si, H. Wu, and Y.-Y. Mo, "MicroRNA-21 targets the tumor suppressor gene tropomyosin 1 (TPM1)," Journal of Biological Chemistry, vol. 282, no. 19, pp. 14328-14336, 2007.

[27] S.-P. Li, H.-X. Su, D. Zhao, and Q.-L. Guan, "Plasma miRNA506 as a prognostic biomarker for esophageal squamous cell carcinoma," Medical Science Monitor, vol. 22, pp. 2195-2201, 2016.

[28] L. Sun, S. Dong, C. Dong et al., "Predictive value of plasma miRNA-718 for esophageal squamous cell carcinoma," Cancer Biomarkers, vol. 16, no. 2, pp. 265-273, 2016.

[29] X. Li, L. Yang, and L.-L. Chen, "The biogenesis, functions, and challenges of circular RNAs," Molecular Cell, vol. 71, no. 3, pp. 428-442, 2018.

[30] L. Chen, S. Zhang, J. Wu et al., "circRNA_100290 plays a role in oral cancer by functioning as a sponge of the miR-29 family," Oncogene, vol. 36, no. 32, pp. 4551-4561, 2017.

[31] W. W. Du, W. Yang, X. Li et al., "A circular RNA circ-DNMT1 enhances breast cancer progression by activating autophagy," Oncogene, vol. 37, no. 44, pp. 5829-5842, 2018.

[32] S. Cao, G. Chen, L. Yan, L. Li, and X. Huang, "Contribution of dysregulated circRNA_100876 to proliferation and metastasis of esophageal squamous cell carcinoma," OncoTargets and Therapy, vol. 11, pp. 7385-7394, 2018.

[33] B. Zheng, Z. Wu, S. Xue et al., "hsa_circRNA_100873 upregulation is associated with increased lymphatic metastasis of esophageal squamous cell carcinoma," Oncology letters, vol. 18, no. 6, pp. 6836-6844, 2019.

[34] X. Hu, D. Wu, X. He et al., "circGSK3 $\beta$ promotes metastasis in esophageal squamous cell carcinoma by augmenting $\beta$-catenin signaling," Molecular Cancer, vol. 18, no. 1, p. 160, 2019.

[35] H. Yang, H. Su, N. Hu et al., "Integrated analysis of genomewide miRNAs and targeted gene expression in esophageal squamous cell carcinoma (ESCC) and relation to prognosis," BMC Cancer, vol. 20, no. 1, p. 388, 2020.

[36] D. Zheng, Y. Ding, Q. Ma et al., "Identification of serum MicroRNAs as novel biomarkers in esophageal squamous cell carcinoma using feature selection algorithms," Frontiers in Oncology, vol. 8, p. 674, 2018.

[37] W. Jiao, J. Zhang, Y. Wei et al., "MiR-139-5p regulates VEGFR and downstream signaling pathways to inhibit the 
development of esophageal cancer," Digestive and Liver Disease, vol. 51, no. 1, pp. 149-156, 2019.

[38] M. Yang, R. Liu, J. Sheng et al., "Differential expression profiles of microRNAs as potential biomarkers for the early diagnosis of esophageal squamous cell carcinoma," Oncology Reports, vol. 29, no. 1, pp. 169-176, 2013.

[39] Z. Jia, Y. Yang, Z. Dengyan et al., "RAP1B, a DVL2 binding protein, activates Wnt/beta-catenin signaling in esophageal squamous cell carcinoma," Gene, vol. 611, pp. 15-20, 2017.

[40] M. Zhang, S. Zhou, L. Zhang et al., "miR-518b is downregulated, and involved in cell proliferation and invasion by targeting Rap1b in esophageal squamous cell carcinoma," FEBS Letters, vol. 586, no. 19, pp. 3508-3521, 2012. 